\title{
A rare cause of acule urinary retention in adolescents: A case of imperforate hymen
}

\author{
Erinna Mohamad Zon, Nik Rafiza Afendi, Nurul Bazilah Mansor, W Fadhlina W Adnan \\ Zon EM, Afendi NR, Mansor NB, et al. A rare cause of acute urinary retention in adolescents: A case of imperforate hymen . Malays Fam \\ Physician. 2021;16(3);112-114. https://doi.org/10.51866/cr1214
}

Keywords:

Imperforate hymen,

haematometrocolpos,

haematocolpos, acute urinary

retention, adolescent

\section{Authors:}

\section{Nik Rafiza Afendi}

(Corresponding author)

MBBCh. BAO Dublin, MMED O\&G

(USM)

Department of Obstetrics \&

Gynaecology, School of Medical

Sciences, Universiti Sains Malaysia

Kelantan, Malaysia

Department of Obstetrics \&

Gynaecology, Hospital Universiti

Sains Malaysia, Kelantan, Malaysia

Email: nikrafiza@usm.my

Erinna Mohamad Zon

MD (USM), MMed (USM)

Department of Obstetrics \&

Gynaecology, School of Medical

Sciences, Universiti Sains Malaysia

Kelantan, Malaysia

Department of Obstetrics \&

Gynaecology, Hospital Universiti

Sains Malaysia, Kelantan, Malaysia

Nurul Bazilah Mansor

MBBCh, BAO Galway

Department of Obstetrics \&

Gynaecology, School of Medical

Sciences, Universiti Sains Malaysia

Kelantan, Malaysia

Department of Obstetrics \&

Gynaecology, Hospital Universiti

Sains Malaysia, Kelantan, Malaysia

\begin{abstract}
Imperforate hymen is a genital outflow abnormality that can occur in females. It can present with various symptoms and is associated with short- and long-term complications that may affect patients' quality of life. Acute urinary retention in adolescents is a rare occurrence. We report a case of delayed diagnosis of imperforate hymen involving multiple visits to the clinic for urinary symptoms and the subsequent development of acute urinary retention. Awareness of this rare presentation is essential since delayed diagnosis is associated with hydronephrosis, endometriosis and infertility in later life.
\end{abstract}

\section{Introduction}

Imperforate hymen is a genital outflow abnormality that can occur in females. It can present with various symptoms and is associated with short- and long-term complications that may affect patients' quality of life. In adolescents, acute urinary retention is a rare occurrence and usually due to an obstructing pelvic or perineal mass. One of the differential diagnoses is colpomenorrhea due to the accumulation of menstrual blood above an imperforate hymen, which leads to distension of the vagina and stretching and lengthening of the urethra. The reason for discussing this case is to highlight clinical presentations and assessments that may help in diagnosing the condition in a primary care setting for early referral to a gynaecologist for intervention.

\section{Case presentation}

An 11-year-old girl presented to the accident and emergency department with a history of sudden onset acute urinary retention and suprapubic pain. She experienced difficulty in passing urine for the previous 2 months, which was associated with dysuria and incomplete voiding. She also complained of cyclical abdominal pain for the past 3 months. She had not yet attained menarche. There was no history of fever or vomiting. Her birth history and developmental history were unremarkable.

She had multiple visits to a private clinic for her symptoms, which were treated as a urinary tract infection (UTI). Although she had completed a few courses of antibiotics, her symptoms persisted.

On examination, she was alert, pink, afebrile and in pain. She was an appropriately built girl with the presence of well-developed secondary sexual characteristics. Her abdomen was soft with mild tenderness at the suprapubic area. There was a palpable mass at the suprapubic region, equivalent to a 16-week uterus size. Her bladder was also palpable. Perineal examination revealed a bulging imperforate hymen exaggerated on Valsalva manoeuvre. Other systemic examinations were unremarkable. The bladder was catheterised and approximately 800 $\mathrm{ml}$ of urine was drained. Pelvic ultrasound revealed a distended uterus and vagina with homogenous and hyperechoic collection. A diagnosis of imperforate hymen with haematometrocolpos was made.

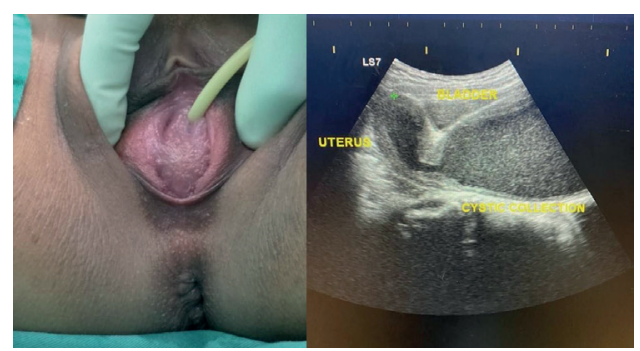

Figure 1. Left: Perineum examination noted imperforate hymen. Right: Ultrasound finding of a distended uterus with cystic collection in the vagina and a ground-glass appearance suggestive of blood collection. 


\author{
W Fadhlina W Adnan \\ MBBCh BAO Galway, MMed O\&G \\ (USM) \\ Department of Obstetrics \& \\ Gynaecology, School of Medical \\ Sciences, Universiti Sains Malaysia \\ Kelantan, Malaysia \\ Department of Obstetrics \& \\ Gynaecology, Hospital Universiti \\ Sains Malaysia, Kelantan, Malaysia
}

Subsequently, the patient underwent a hymenectomy where a cruciate incision was made over the hymen. Approximately $300 \mathrm{ml}$ of thick chocolate brown-coloured blood was drained.

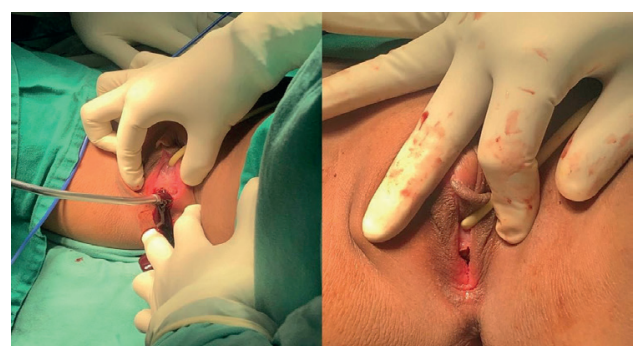

Figure 2. Right: Old menstrual blood drained following hymenectomy. Left: Post hymenectomy.

Oral analgesics and antibiotics were prescribed post-operatively. The patient made an uneventful recovery and was well at the 1-month follow-up. She also started experiencing regular menses and had no further urinary problems.

\section{Discussion:}

Imperforate hymen is one of the most common obstructive lesions of the female genital tract. It is the result of failed embryologic canalisation of the most caudal portion of the vaginal plate at its juncture with the urogenital sinus. ${ }^{1}$ The incidence of imperforate hymen is 1 in 1000 to 1 in 2000 women. ${ }^{2,3,4}$

It is rare to detect an imperforate hymen in infancy. Most cases of imperforate hymen are found at puberty. ${ }^{4}$ Around the age of menarche, an adolescent girl usually presents with amenorrhoea and cyclical abdominal or pelvic pain. Less common symptoms include back pain and constipation. If the accumulation of blood and cellular debris is large, it can compress the urethra and bladder, thereby giving rise to symptoms of urinary tract obstruction such as dysuria, urinary frequency and feelings of incomplete voiding. These symptoms can be mistaken as UTI symptoms, which explains why some patients are misdiagnosed as having a UTI. ${ }^{5}$ Delayed diagnosis will lead to acute urinary retention, as in the case of our patient. The incidence of acute urinary retention in patients with imperforate hymen is rare. ${ }^{6}$

Assessing the history of pubertal development and examining secondary sexual characteristics are important in diagnosing adolescent girls. Menarche typically occurs within 2-3 years after thelarche (breast budding), at Tanner stage IV breast development. ${ }^{7}$ Imperforate hymen should be considered in all adolescent girls with breast development of at least Tanner stage III complaining of cyclical pelvic pain, back pain or urinary symptoms that have not attained menarche.

A simple clinical examination of the external genitalia usually reveals a tense, bluish bulging membrane that is pathognomonic of imperforate hymen.

A bedside pelvic ultrasound is sufficient to reveal blood collection in the vagina (haematocolpos) that may even extend to the uterus (haematometrocolpos) and (rarely) involve the fallopian tubes. ${ }^{8}$

Early recognition and treatment of the problem are required to prevent longterm complications for endometriosis and infertility. ${ }^{9}$ Treatment of imperforate hymen involves the simple surgical procedure of hymenectomy.

\section{Conclusion}

Imperforate hymen is a diagnosis that can easily be made with a careful history, a complete examination and a high index of suspicion. Presentations of imperforate hymen vary from a classical presentation of amenorrhoea and cyclical abdominal pain or pelvic pain to symptoms of compression due to massive haematocolpos or haematometrocolpos collection resulting in symptoms of urinary retention, acute urinary retention, constipation or back pain. Symptoms of urinary retention mimic the symptoms of UTI; thus, these patients can be initially misdiagnosed as having a recurrent UTI. Early recognition of this condition will avoid complications such as acute urinary retention or endometriosis.

\section{Acknowledgements}

None.

\section{Conflicts of interest}

The authors declare that they have no conflicts of interest.

Patient's consent for the use of images and content for publication:

Verbal and written consent obtained. 


\section{How does this paper make a difference to general practice?}

- Cyclical abdominal pain in girls who had not yet attained their menarche is a classical symptom suggestive of outflow tract obstruction.

- Symptoms of recurrent UTI or acute urinary retention is a rare presentation of imperforate hymen.

- Knowledge of the pubertal stages is essential in detecting an atypical presentation of imperforate hymen, and an assessment of secondary sexual characteristics should be performed on all adolescent girls presented to the clinic with abdominal pain.

- Bedside ultrasound is important in the assessment of patients with pelvic/abdominal symptoms. This can be easily performed since most health clinics/GP practices are equipped with an ultrasound machine.

\section{What is new in this case report compared to the previous literature?}

Acute urinary retention in children is rare. Acute urinary retention presented due to imperforate hymen is even rarer. Although a few similar cases have been published before, many remain unaware of atypical presentations of imperforate hymen (e.g., acute urinary retention). The lack of awareness regarding atypical presentations of imperforate hymen must be addressed to prevent delayed or missed diagnoses leading to long-term complications.

\section{What are the implications for patients?}

The accumulation of blood in the vagina (and subsequently in the uterus) will cause cyclical pain for patients. The undiagnosed or delayed diagnosis of imperforate hymen causes unnecessary pain, which will disrupt patients' studies and social interactions. It can also cause acute urinary retention (as in our case) due to compression of the urethra, which may lead to hydronephrosis. ${ }^{10,11}$ Furthermore, delayed diagnosis of imperforate hymen will increase the risk of menstrual blood backflow into the peritoneal cavity, which may increase the risk of endometriosis and infertility in later life. ${ }^{12}$

\section{References}

1. Coppola L. Unique case of imperforate hymen. J Pediatr Adolesc Gynecol. 2016;29(1):e1-3.

2. ASRM Practice Committee.Current evaluation of amenorrhoea. Fertility and Sterility 2008;90(3): S219-25.

3. Dietrich JE, Millar DM, Quint EH. Obstructive reproductive tract anomalies. J Pediatr Adolesc Gynecol. 2014;27(6):396-402.

4. Grimstad F, Strickland J, Dowlut-McElroy T. Management and prevention of postoperative complications in a neonate with a symptomatic imperforate hymen. J Pediatr Adolesc Gynecol. 2019;32(4):429-31.

5. Abraham C. Imperforate hymen causing hematocolpos and urinary retention. J Emerg Med. 2019;57(2):238-40.
6. Patoulias I, Prodromou K, Kallergis K, et al. Acute urinary retention due to hematocolpos: Report of two cases. J Pediatr Surg Case Reports. 2013;1(8):189-91.

7. The American College of Obstetricians and Gynecologists. Committee opinion summary menstruation in girls and adolescents: Using the menstrual cycle as a vital sign. Obstet Gynecol. 2015;126(640):691-2.

8. Amponsah-Manu F, Ssentongo P, Arkorful $\mathrm{T}$, et al. Imperforate hymen and leaking hematosalpinx mimicking acute appendicitis: A report of a rare case and a review of literature. Int J Surg Case Rep. 2019;63:69-74.

9. Ayrim AA, Gözdemİr E, Turhan NÖ, et al. Acute urinary retention associated with an imperforate hymen and hematocolpos. Gynaecol Obstet Reprod Med. 2009;15(2):105-7.
10. Loscalzo I, Catapano M, Loscalzo J, et al. Clinical communications: Imperforate hymen with bilateral hydronephrosis. Science. 1995;13(3):337-9.

11. Niang I, Diouf KN, Thiam M, et al. Late diagnosis of imperforate hymen with hematometrocolpos and bilateral hydronephrosis of a horseshoe kidney. Radiol Case Reports. 2020;15(11):2217-20.

12. Adolescent endometriosis: An update. J Pediatr Adolesc Gynecol. 2020;33(2):112-9. 